Detecting Autocatalytic, Self-sustaining sets in Chemical Reaction Systems

Wim Hordijk and Mike Steel

\author{
Department of Mathematics and Statistics \\ University of Canterbury \\ Private Bag 4800 \\ Christchurch, New Zealand
}




\title{
DETECTING AUTOCATALYTIC, SELF-SUSTAINING SETS IN CHEMICAL REACTION SYSTEMS
}

\author{
Wim Hordijk and Mike Steel \\ Biomathematics Research Centre \\ University of Canterbury \\ Christchurch, New Zealand \\ $\{$ W.Hordijk, M.Steel\}@math. canterbury.ac.nz
}

\begin{abstract}
The ability of systems of molecular reactions to be simultaneously autocatalytic and sustained by some ambient 'food source' of simple molecules may have been an essential step in the origin of life. In this paper we first describe a polynomial-time algorithm that determines whether any given set of molecules, reactions and catalysations contains a subsystem that is both autocatalytic and able to be sustained from a given subset of the molecules. We also describe some combinatorial properties of this algorithm, and show how it can be used to find irreducible auto-catalysing and sustaining subsystems. In the second part of the paper we use the algorithm to investigate random catalytic networks - in particular a model described by Kauffman. Using simulations and some analytic techniques we investigate the rate of catalysis that is required for the emergence of autocatalytic and sustaining subsystems.
\end{abstract}

Keywords - Artificial life, autocatalysis, random combinatorial structures.

\section{INTRODUCTION}

The origin of life still remains an elusive problem. Two main paradigms currently exist: the RNA-first theory and the protein-first theory (see, e.g., [11, 8]). The former theory (RNA-first) maintains that life started with self-reproducing RNA molecules, which replicate by virtue of template complementarity. These molecules then evolved to encode (genetic) information that is translated into proteins. The main drawback of this theory is that replication (even template-based) is difficult, or at least very slow, to achieve without catalysts. Although it has been shown that some RNA has catalytic capability, this is only very limited and quite specific. Thus, the appearance of an RNA molecule that catalyses its own replication (or even that of others) seems highly unlikely.

In the other main theory (protein-first) it is assumed that life started as collectively self-reproducing sets of catalytic polymers (proteins). Since proteins form easily from freely available amino acids, and even small proteins have catalytic capabilities (often quite general), this theory may seem more plausible. However, proteins do not have a template structure, and thus it is hard to envision how they could have replicated easily without the aid of a genetic code. 
An alternative theory, which allows both scenarios to occur concurrently, even cooperatively, is that of autocatalytic sets of molecules (including RNA and proteins, and possibly others). The idea is that molecules engage in catalysed chemical reactions, creating or transforming into other molecules. It has been argued that in sufficiently complex chemical reaction systems, an autocatalytic set will emerge spontaneously, i.e., a subset of molecules and reactions where each molecule is created by at least one reaction from this set, and each reaction is catalysed by at least one molecule from the set ('catalytic closure') $[5,7,8]$.

Here, we formally investigate this probability of autocatalytic sets arising in chemical reaction systems in more detail, both theoretically and numerically. We present a general, polynomial-time algorithm for detecting, in any given chemical reaction system, autocatalytic sets that can be sustained from a suitable 'food' source. We then apply our algorithm to perform numerical simulations, combined with some analytic techniques, to investigate Kauffman's simple abstract origin of life model involving large numbers of molecules randomly catalysing the ligation and cleavage of other molecules $[7,8]$. Kauffman claimed that life-like subsystems ('connected, reflexively autocatalytic' sets) must spontaneously arise with high probability once the number of molecule types becomes sufficiently large, a conclusion that was subsequently criticised by Lifson [9], and partially resolved in [12].

The probability of such autocatalytic sets occurring depends largely on the probability $p$ that a molecule catalyses any given reaction. In Kauffman's model, this probability remains constant, even if the total number of possible reactions increases (e.g., by virtue of having more and more molecule types). In other words, the average number of reactions that a molecule catalyses increases rapidly with an increasing number of molecule types. It is intuitively clear that, given enough molecule types, an autocatalytic network will then occur with high probability. However, in Lifson's interpretation of Kauffman's model, this probability of catalysation $p$ is inversely proportional to the total number of possible reactions, and thus the average number of reactions that a molecule catalyses remains constant with an increasing number of molecule types. In this case, it is not clear whether an autocatalytic set will arise at all, and for $p$ low enough it is almost certain it will not [12].

What is of interest here, is what happens in between these two extreme cases (constant or inverse proportional catalysation probabilities). In particular, where does the transition occur whereby the existence of an autocatalytic set changes from being highly unlikely to being almost certain? And, furthermore, what does this transition look like? In this paper we provide a theorem that shows that an intermediate degree of catalysation (between the two extremes) suffices for there to be, on average, a large number of self-sustaining autocatalytic systems (in line with a conjecture from [12]). We then provide numerical results generated by an implementation of our algorithm as applied to Kauffman's model, which shed additional light on the occurrence and shape of the transition between the two extreme cases. 
The paper is organised as follows. Section 2 formally introduces catalytic reaction systems, and section 3 defines the notion of autocatalytic sets generated by a food source (called $R A F$ sets here). Section 4 then proves some basic properties of RAF sets, after which section 5 introduces a pair of operations that can be applied to any catalytic reaction system to reduce its size while maintaining these basic properties. Section 6 then states our main result and shows how the reduction rules can be used to find whether a catalytic reaction system contains an RAF set. Section 7 presents the complete algorithm for finding RAF sets and shows that it has a polynomial running time. Section 8 then provides an overview of random catalytic reaction systems and formalizes Kauffman's model, while the results of our simulations are presented in section 9. Finally, section 10 summarises our main conclusions.

\section{Catalytic REACTION SYSTEMS}

In this section, we review the notion of catalytic reaction systems and reaction graphs.

\section{Definitions:}

- Let $X$ denote a set of molecules. A reaction is an ordered pair $r=(A, B)$ where $A, B \subset X$, and $A \cap B=\emptyset$. If $A=\left\{a_{1}, \ldots a_{s}\right\}$ and $B=\left\{b_{1}, \ldots, b_{t}\right\}$ then $r$ represents an admissible chemical reaction in which the molecules in $A$ (the 'reactants') combine, in certain proportions, to produce the molecules in $B$ (the 'products'), written

$$
n_{1} a_{1}+\cdots+n_{s} a_{s} \rightarrow n_{1}^{\prime} b_{1}+\cdots+n_{t}^{\prime} b_{t}
$$

where $n_{i}$ and $n_{i}^{\prime}$ are positive integers for all $i$. Note that a reversible chemical reaction such as $n_{1} a_{1}+\cdots+n_{s} a_{s} \rightleftharpoons n_{1}^{\prime} b_{1}+\cdots+n_{t}^{\prime} b_{t}$ can be described by specifying the pair $(A, B)$ and $(B, A)$.

- Let $F$ (for 'food') denote a distinguished subset of $X$.

- Let $\mathcal{R}$ be a set of allowable reactions. A catalysation is a pair $(x, r)$ where $x \in X, r \in \mathcal{R}$, denoting that molecule $x$ catalyses reaction $r$. Let $C \subseteq X \times \mathcal{R}$ be a set of catalysations.

- For $r=(A, B) \in \mathcal{R}$, let $\rho(r)=A, \pi(r)=B$, and for a subset $\mathcal{R}^{\prime}$ of $\mathcal{R}$, set $\rho\left(\mathcal{R}^{\prime}\right)=\cup_{r \in \mathcal{R}^{\prime}} \rho(r), \pi\left(\mathcal{R}^{\prime}\right)=\cup_{r \in \mathcal{R}^{\prime}} \pi(r)$ and $\operatorname{supp}\left(\mathcal{R}^{\prime}\right)=\rho\left(\mathcal{R}^{\prime}\right) \cup \pi\left(\mathcal{R}^{\prime}\right)$. Thus, $\operatorname{supp}\left(\mathcal{R}^{\prime}\right)$ (the 'support' of $\mathcal{R}^{\prime}$ ) is the set of all molecules that are involved in at least one reaction in $\mathcal{R}^{\prime}$.

- The triple $(X, \mathcal{R}, C)$ will be called a catalytic reaction system (over $F$ ), or CRS (over $F$ ).

Following [10], [3](p.262ff) we may represent the catalytic reaction system $\mathcal{Q}=$ $(X, \mathcal{R}, C)$ as a directed graph on vertex set $X \cup \mathcal{R}$, and with two types or arcs, described as follows. For each $r=(A, B) \in \mathcal{R}$, we place arcs from each element $a \in A$ to $r$ and from $r$ to each element $b \in B$. We will refer to these as reaction arcs. We also place an arc from any $x \in X$ to any $r \in R$ precisely when $(x, r) \in C$ and refer to these as catalytic arcs. 
As an example, consider the following four reactions:

$$
\begin{aligned}
& r_{1}: a+b \stackrel{d}{\rightarrow} c \\
& r_{2}: b+c \stackrel{a}{\rightarrow} d \\
& r_{3}: c+d \rightarrow e+f \\
& r_{4}: a+e \stackrel{f}{\rightarrow} g
\end{aligned}
$$

So, $X=\{a, b, c, d, e, f, g\}, \mathcal{R}=\left\{r_{1}, r_{2}, r_{3}, r_{4}\right\}$ and $C=\left\{\left(d, r_{1}\right),\left(a, r_{2}\right),\left(f, r_{4}\right)\right\}$. Let the food set be $F=\{a, b\}$, for example. Then $\mathcal{Q}=(X, \mathcal{R}, C)$ is a catalytic reaction system over $F$. This CRS $\mathcal{Q}$ can be represented by the catalytic reaction graph shown in Fig. 1. In this graph, black vertices represent molecules (the set $X$ ), white vertices represent reactions (the set $\mathcal{R}$ ), solid lines represent reaction arcs, and dashed lines represent catalytic arcs.

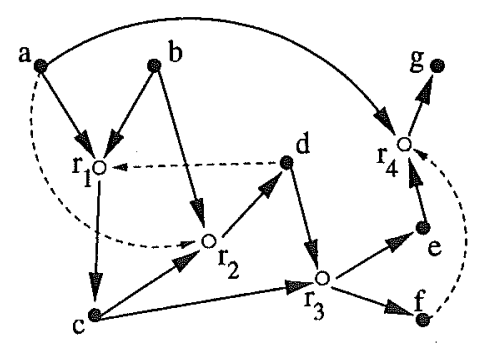

FIGURE 1. An example of a catalytic reaction graph.

\section{Autocatalytic Sets generated BY A FOod SOURCe}

Given a subset $\mathcal{R}^{\prime}$ of $\mathcal{R}$, and a subset $X^{\prime}$ of $X$, define the closure of $X^{\prime}$ relative to $\mathcal{R}^{\prime}$, denoted $\operatorname{cl}_{\mathcal{R}^{\prime}}\left(X^{\prime}\right)$ to be the (unique) minimal subset $W$ of $X$ that contains $X^{\prime}$ and that satisfies the condition that, for each reaction $(A, B)$ in $\mathcal{R}^{\prime}$,

$$
A \subseteq X^{\prime} \cup W \Longrightarrow B \subseteq W .
$$

Informally, $\mathrm{cl}_{\mathcal{R}^{\prime}}\left(X^{\prime}\right)$ is $X^{\prime}$ together with all molecules that can be constructed from $X^{\prime}$ by the repeated application of reactions in $\mathcal{R}^{\prime}$. Note that $\mathrm{cl}_{\mathcal{R}^{\prime}}\left(X^{\prime}\right)$ is well defined and is a subset of $\pi\left(\mathcal{R}^{\prime}\right) \cup X^{\prime}$ since $\pi\left(\mathcal{R}^{\prime}\right) \cup X^{\prime}$ satisfies (1) and the collection of subsets of $W \subseteq X$ satisfying (1) is closed under intersection.

Given a CRS $\mathcal{Q}=(X, \mathcal{R}, C)$, a nonempty subset $\mathcal{R}^{\prime}$ of $\mathcal{R}$ is said to be:

- reflexively autocatalytic (RA) for $\mathcal{Q}$ if

for all $r \in \mathcal{R}^{\prime}$ there exists an $x \in \operatorname{supp}\left(\mathcal{R}^{\prime}\right):(x, r) \in C$,

- F-generated if

$$
\rho\left(\mathcal{R}^{\prime}\right) \subseteq \operatorname{cl}_{\mathcal{R}^{\prime}}(F),
$$

- reflexively autocatalytic and $F$-generated (RAF) for $\mathcal{Q}$ if $\mathcal{R}^{\prime}$ is $\mathrm{RA}$ for $\mathcal{Q}$ and generated by $F$. In this case we refer to $\mathcal{R}^{\prime}$ as an $R A F$ set for $\mathcal{Q}$. 
Informally, a set of reactions $\mathcal{R}^{\prime}$ is RAF if every reaction is catalysed by at least one molecule involved in a reaction in $\mathcal{R}^{\prime}$, and every reactant in $\mathcal{R}^{\prime}$ can be constructed from the food set $F$ by successive applications of reactions from $\mathcal{R}^{\prime}$. It thus captures the abstract idea of 'life' as an auto-catalysing system able to sustain itself by using a suitable food source.

As an example, the subset $\mathcal{R}^{\prime}=\left\{r_{1}, r_{2}\right\}$ from the previous section forms an RAF set, and is shown in Fig. 2. Each reaction in $\mathcal{R}^{\prime}$ is catalysed by an element in $\operatorname{supp}\left(\mathcal{R}^{\prime}\right)$, and all reactants can be constructed from $F$ by one or more applications of $r_{1}$ and $r_{2}$.

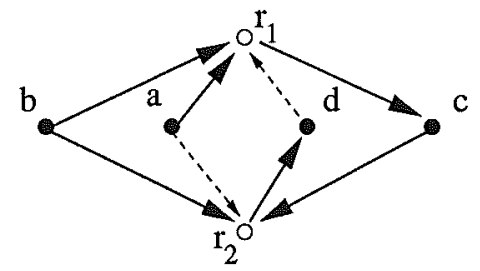

Figure 2. An example of an RAF set with two reactions, with $F=\{a, b\}$.

In [12] an RAF is referred to as a CRA (connected, reflexively autocatalytic). However, we have chosen RAF here to emphasise the dependence on $F$.

\section{Properties of RAF SETS}

In this section we present some lemmas that provide useful information on the structure of RAF sets.

Lemma 4.1. Consider a catalytic reaction system $\mathcal{Q}=(X, \mathcal{R}, C)$ over $F$, and two subsets $\mathcal{R}_{1}, \mathcal{R}_{2}$ of $\mathcal{R}$. Then,

(i) If $\mathcal{R}_{1}$ and $\mathcal{R}_{2}$ are both $R A$ for $\mathcal{Q}$ (respectively $F-$ generated) then $\mathcal{R}_{1} \cup \mathcal{R}_{2}$ is $R A$ for $\mathcal{Q}$ (respectively $F$-generated).

(ii) If $\mathcal{R}_{1}$ and $\mathcal{R}_{2}$ are both $R A F$ for $\mathcal{Q}$ then $\mathcal{R}_{1} \cup \mathcal{R}_{2}$ is $R A F$ for $\mathcal{Q}$.

Proof. The result for RA in part (i) is clear from the definition. If $\mathcal{R}_{1}$ and $\mathcal{R}_{2}$ are both $F$-generated, then

$$
\rho\left(\mathcal{R}_{1} \cup \mathcal{R}_{2}\right)=\rho\left(\mathcal{R}_{1}\right) \cup \rho\left(\mathcal{R}_{2}\right) \subseteq \operatorname{cl}_{\mathcal{R}_{1}}(F) \cup \operatorname{cl}_{\mathcal{R}_{2}}(F) \subseteq \operatorname{cl}_{\left(\mathcal{R}_{1} \cup \mathcal{R}_{2}\right)}(F)
$$

and so $R_{1} \cup R_{2}$ is $F$-generated.

Part (ii) follows immediately from part (i).

An immediate consequence of this lemma is the following. 
Corollary 4.2. If the catalytic reaction system $\mathcal{Q}=(X, \mathcal{R}, C)$ over $F$ has an $R A(F)$ set, then it has a maximal $R A(F)$ set, namely

$$
\bigcup\left\{\mathcal{R}^{\prime}: \mathcal{R}^{\prime} \subseteq \mathcal{R} \text {, and } \mathcal{R}^{\prime} \text { is an } R A(F) \text { set for } \mathcal{Q}\right\}
$$

Lemma 4.3. Given a catalytic reaction system $\mathcal{Q}, \mathcal{R}^{\prime}$ is $F$-generated if and only if

$$
\mathrm{cl}_{\mathcal{R}^{\prime}}(F)=F \cup \operatorname{supp}\left(\mathcal{R}^{\prime}\right)
$$

Proof. Suppose that $\mathcal{R}^{\prime}$ is $F$-generated. Then $\rho\left(\mathcal{R}^{\prime}\right) \subseteq \operatorname{cl}_{\mathcal{R}^{\prime}}(F)$, which implies that $\pi\left(\mathcal{R}^{\prime}\right) \subseteq \operatorname{cl}_{\mathcal{R}^{\prime}}(F)$. Thus, since $F \subseteq \operatorname{cl}_{\mathcal{R}^{\prime}}(F),(2)$ holds. Conversely, suppose that (2) holds. Then $\rho\left(\mathcal{R}^{\prime}\right) \subseteq \operatorname{cl}_{\mathcal{R}^{\prime}}(F)$ and so $\mathcal{R}^{\prime}$ is $F$-connected.

We next define a partial order on catalytic reaction systems as follows: If $\mathcal{Q}_{1}=$ $\left(X_{1}, \mathcal{R}_{1}, C_{1}\right)$ and $\mathcal{Q}_{2}=\left(X_{2}, \mathcal{R}_{2}, C_{2}\right)$, then we write $\mathcal{Q}_{1} \leq \mathcal{Q}_{2}$ if and only if $X_{1} \subseteq$ $X_{2}, \mathcal{R}_{1} \subseteq \mathcal{R}_{2}, C_{1} \subseteq C_{2}$. Furthermore, we define a grading on this poset by setting

$$
l(X, \mathcal{R}, C)=|X|+|\mathcal{R}|
$$

Clearly, $\mathcal{Q}_{1} \leq \mathcal{Q}_{2}$ implies that $l\left(\mathcal{Q}_{1}\right) \leq l\left(\mathcal{Q}_{2}\right)$. The next result is an immediate consequence of the definitions.

Lemma 4.4. Suppose that $\mathcal{Q}_{1}$ and $\mathcal{Q}_{2}$ are catalytic reaction systems over $F$ and that $\mathcal{Q}_{1} \leq \mathcal{Q}_{2}$. If $\mathcal{R}^{\prime}$ is $R A(F)$ for $\mathcal{Q}_{1}$ then $\mathcal{R}^{\prime}$ is $R A(F)$ for $\mathcal{Q}_{2}$.

\section{REDUCTION RULES}

In this section we introduce a pair of reduction rules which can be applied to any catalytic reaction system $\mathcal{Q}=(X, \mathcal{R}, C)$. These rules operate as follows.

(R1) For any $r \in \mathcal{R}$ with $\{x \in \operatorname{supp}(\mathcal{R}):(x, r) \in C\}=\emptyset$, delete $r$ from $\mathcal{R}$.

(R2) For any $r \in \mathcal{R}$ for which $\rho(r) \nsubseteq \mathrm{cl}_{\mathcal{R}}(F)$, delete $r$ from $\mathcal{R}$.

Let $\gamma(\mathcal{R}) \subseteq \mathcal{R}$ denote a set of reactions obtained by repeated applications of (R1) to $(X, \mathcal{R}, \bar{C})$ until no further reductions can be made, and let $\delta(\mathcal{R}) \subseteq \mathcal{R}$ denote a set of reactions obtained by repeated applications of $(\mathrm{R} 2)$ to $(X, \mathcal{R}, C)$ until no further reductions can be made.

Lemma 5.1. Consider a catalytic reaction system $\mathcal{Q}=(X, \mathcal{R}, C)$, and suppose $\mathcal{R}^{\prime} \subseteq \mathcal{R}$ is a non-empty set of reactions.

(i) $\mathcal{R}^{\prime}$ is an $R A$ set for $\mathcal{Q}$ if and only if $\gamma\left(\mathcal{R}^{\prime}\right)=\mathcal{R}^{\prime}$.

(ii) $\mathcal{R}^{\prime}$ is $F$-generated, if and only if $\delta\left(\mathcal{R}^{\prime}\right)=\mathcal{R}^{\prime}$.

(iii) If $\gamma(\mathcal{R})=\emptyset$ then there is no $R A$ set for $\mathcal{Q}$, while if $\gamma(\mathcal{R}) \neq \emptyset$ then $\gamma(\mathcal{R})$ is the maximal $R A$ set for $\mathcal{Q}$. In particular, $\gamma(\mathcal{R})$ is independent of the order by which (R1) is applied to reactions.

(iv) If $\delta(\mathcal{R})=\emptyset$ there is no $F$-generated set in $\mathcal{Q}$, while if $\delta(\mathcal{R}) \neq \emptyset$ then $\delta(\mathcal{R})$ is the maximal $F$-generated set in $\mathcal{Q}$. In particular, $\delta(\mathcal{R})$ is independent of the order by which (R2) is applied to reactions. 
Proof. Parts (i) and (ii) follow directly from the definition of RA and $F$-generated.

For part (iii), suppose that $\mathcal{R}_{1}$ is RA for $\mathcal{Q}$ where $\mathcal{R}_{1} \subseteq \mathcal{R}$. Now, suppose that rule (R1) applies to some reaction $r \in \mathcal{R}$ - that is, there is no $x \in \operatorname{supp}(\mathcal{R})$ for which $(x, r) \in C$. Since $\mathcal{R}_{1} \subseteq \mathcal{R}$, we have $\operatorname{supp}\left(\mathcal{R}_{1}\right) \subseteq \operatorname{supp}(\mathcal{R})$, and so there is no $x \in \operatorname{supp}\left(\mathcal{R}_{1}\right)$ for which $(x, r) \in C$. Thus, since $\mathcal{R}_{1}$ is RA it follows that $r \notin \mathcal{R}_{1}$. In particular $\mathcal{R}_{1} \subseteq \mathcal{R}-\{r\}$. By induction it follows that $\mathcal{R}_{1} \subseteq \gamma(\mathcal{R})$. Since this holds for all choices of $\mathcal{R}_{1}$ that are RA sets for $\mathcal{Q}$ it follows that $\gamma(\mathcal{R})$ contains all RA sets for $\mathcal{Q}$. Consequently, if $\gamma(\mathcal{R})=\emptyset$ then $\mathcal{Q}$ has no RA set while if $\gamma(\mathcal{R}) \neq \emptyset$, then since $\gamma(\mathcal{R})$ is itself an RA set for $\mathcal{Q}$ (by part (i), since $\gamma(\gamma(\mathcal{R}))=\gamma(\mathcal{R})$ ), it follows that $\gamma(\mathcal{R})$ is the maximal RA set for $\mathcal{Q}$.

The proof of part (iv) is similar to part (iii) but for completeness we provide it here. Suppose that $\mathcal{R}_{1}$ is $F$-generated, where $\mathcal{R}_{1} \subseteq \mathcal{R}$. Now, suppose that rule (R2) applies to some reaction $r \in \mathcal{R}$ - that is, $\rho(r) \nsubseteq \mathrm{cl}_{\mathcal{R}}(F)$. Since $\mathcal{R}_{1} \subseteq \mathcal{R}$, we have $\operatorname{cl}_{\mathcal{R}_{1}}(F) \subseteq \mathrm{cl}_{\mathcal{R}}(F)$, and so $\rho \nsubseteq \mathrm{cl}_{\mathcal{R}_{1}}(F)$. Thus, since $\mathcal{R}_{1}$ is $F$-generated it follows that $r \notin \mathcal{R}_{1}$. In particular, $\mathcal{R}_{1} \subseteq \mathcal{R}-\{r\}$. By induction it follows that $\mathcal{R}_{1} \subseteq \delta(\mathcal{R})$. Thus $\delta(\mathcal{R})$ contains all $F$-generated sets. Consequently, if $\delta(\mathcal{R})=\emptyset$ then $\mathcal{Q}$ has no $F$-generated set, while if $\delta(\mathcal{R}) \neq \emptyset$, then since $\delta(\mathcal{R})$ is itself $F$ generated (by part (ii), since $\delta(\delta(\mathcal{R}))=\delta(\mathcal{R})$ ), it follows that $\delta(\mathcal{R})$ is the maximal $F$-generated set for $\mathcal{Q}$.

\section{Finding Recursively autocatalytic (AND RAF) SETS}

Using the operations $\gamma$ and $\delta$ as introduced in the previous section, we can now construct a decreasing (in size) and nested sequence $\mathcal{R}=\mathcal{R}_{1}, \mathcal{R}_{2}, \ldots$ of subsets of $\mathcal{R}$ where

Let $\mathcal{R}_{\infty}=\bigcap_{i \geq 1} \mathcal{R}_{i}$

$$
\mathcal{R}_{i+1}=\delta\left(\gamma\left(\mathcal{R}_{i}\right)\right)
$$

Given a catalytic reaction system $\mathcal{Q}=(X, \mathcal{R}, C)$, and a subset $\mathcal{R}^{\prime}$ of $R$, we say that $\mathcal{R}^{\prime}$ is an irreducible RAF for $\mathcal{Q}$ if $\mathcal{R}^{\prime}$ is RAF for $\mathcal{Q}$, but no proper subset of $\mathcal{R}^{\prime}$ has this property.

We can now state our main result.

Theorem 6.1. A catalytic reaction system $\mathcal{Q}=(X, \mathcal{R}, C)$ has an $R A F$ set if and only if $\mathcal{R}_{\infty}$ is non-empty, in which case $\mathcal{R}_{\infty}$ is the maximal $R A F$ set for $\mathcal{Q}$. Furthermore, there exists a polynomial-time (in $l(\mathcal{Q})$ ) algorithm for computing $\mathcal{R}_{\infty}$ and, when this is non-empty, finding an irreducible $R A F$ set for $\mathcal{Q}$.

Proof. Suppose that $\mathcal{R}_{\infty}=\emptyset$ and that there exists a nonempty set $\mathcal{R}^{\prime}$ that is an $\mathrm{RAF}$ set for $\mathcal{Q}$. We will derive a contradiction. First select any value of $i \geq 1$ for which $\mathcal{R}^{\prime} \subseteq \mathcal{R}_{i}$ - this is always possible since $\mathcal{R}_{1}=\mathcal{R}$. Then $\mathcal{R}^{\prime}$ is an RA for $\left(X, \mathcal{R}_{i}, C\right)$. It then follows from Lemma 5.1 (iii) that

$$
\mathcal{R}^{\prime} \subseteq \gamma\left(\mathcal{R}_{i}\right)
$$

Furthermore, since $\mathcal{R}^{\prime}$ is $F$-connected we have $\mathcal{R}^{\prime}=\delta\left(\mathcal{R}^{\prime}\right)$ by Lemma 5.1(ii). Now, by $(3), \delta\left(\mathcal{R}^{\prime}\right) \subseteq \delta\left(\gamma\left(R_{i}\right)\right)=\mathcal{R}_{i+1}$, and so $\mathcal{R}^{\prime} \subseteq \mathcal{R}_{i+1}$. By induction, $\mathcal{R}^{\prime} \subseteq \mathcal{R}_{n}$ for 
all $n \geq i$. However, since $\mathcal{R}_{\infty}=\emptyset$ there exists a value $m$ for which $\mathcal{R}_{n}=\emptyset$ for all $n \geq m$, which contradicts the assumption that $\emptyset \neq \mathcal{R}^{\prime} \subseteq \mathcal{R}_{n}$ for all $n>i$.

Now suppose that $\mathcal{R}_{\infty}$ is nonempty. Then there exists a finite value of $n \geq 1$ for which $\mathcal{R}_{n}=\mathcal{R}_{n+1}$ in which case $\mathcal{R}_{\infty}=\mathcal{R}_{n}$. Now, since $\mathcal{R}_{n}=\delta\left(\gamma\left(\mathcal{R}_{n}\right)\right)$ and since $\gamma\left(\mathcal{R}_{n}\right) \subseteq \mathcal{R}_{n}$, and $\delta\left(\mathcal{R}^{\prime}\right) \subseteq \mathcal{R}^{\prime}$ (for any $\mathcal{R}^{\prime}$, in particular $\mathcal{R}^{\prime}=\gamma\left(\mathcal{R}_{n}\right)$ ), it follows that:

$$
\gamma\left(\mathcal{R}_{n}\right)=\mathcal{R}_{n} \text { and } \delta\left(\mathcal{R}_{n}\right)=\mathcal{R}_{n}
$$

and Lemma 5.1 (parts (i) and (ii)) implies that $\mathcal{R}_{n}\left(=\mathcal{R}_{\infty}\right)$ is both an RA for $\mathcal{Q}$ and is $F$-generated. Consequently, $\mathcal{R}_{\infty}$ is an RAF set for $\mathcal{Q}$.

In the next section an algorithm for computing $\mathcal{R}_{\infty}$ is described. It is shown there that the (worst case) running time of this algorithm is $O\left(|X||\mathcal{R}|^{3}\right)$, i.e., polynomial in $l(\mathcal{Q})$.

We complete the proof by showing how to construct an irreducible RAF set when $\mathcal{R}_{\infty} \neq \emptyset$. First, form a finite decreasing nested series $\mathcal{R}^{1}, \mathcal{R}^{2}, \ldots$ of subsets of $\mathcal{R}_{\infty}$ as follows. Set $\mathcal{R}^{1}=\mathcal{R}_{\infty}$. To construct $\mathcal{R}^{i+1}$ from $\mathcal{R}^{i}$, select any element $r \in \mathcal{R}^{i}$ for which $\mathcal{R}^{i}-\{r\}$ has an RAF set (i.e. $\left(\mathcal{R}^{i}-\{r\}\right)_{\infty} \neq \emptyset$ ), and let $\mathcal{R}^{i+1}=\mathcal{R}^{i}-\{r\}$. If $\mathcal{R}^{i}$ contains no element $r$ for which $R^{i}-\{r\}$ is an RAF set, then $\mathcal{R}^{i}$ is an irreducible RAF set. Note that if $\left(\mathcal{R}^{i}-\{r\}\right)_{\infty}=\emptyset$, then for any value $j \geq i$ we have $\left(\mathcal{R}^{j}-\{r\}\right)_{\infty}=\emptyset$, and so each element of $\mathcal{R}^{1}$ is considered at most once in the formation of the series $\mathcal{R}^{i}$. Since the determination of whether or not $\left(\mathcal{R}^{i}-\{r\}\right)_{\infty}=\emptyset$ takes $O\left(|X \| \mathcal{R}|^{3}\right)$ time (at most), the running time to determine an irreducible RAF, if one exists, is bounded by $O\left(|X||\mathcal{R}|^{4}\right)$.

\section{The RAF ALGORITHM}

We now present the polynomial-time algorithm for computing $\mathcal{R}_{\infty}$. The algorithm consists of three separate subroutines that are called sequentially and repeatedly. All three subroutines assume the existence of a set $X$ of molecule types, a set $\mathcal{R}$ of reactions, a set $C$ of catalysations, and a set $W$ representing the closure of the food set $F$ relative to $\mathcal{R}$. The subroutines assume $X, F$, and $C$ to be fixed, but will change the contents of the sets $W$ and $\mathcal{R}$.

The first subroutine, ReduceToRA, implements the $\gamma(\mathcal{R})$ function, and reduces a given reaction set $\mathcal{R}$ to an RA set. In other words, the ReduceToRA routine repeatedly applies the reduction rule $R 1$ until no more reductions can be made. The pseudocode for this subroutine is given in Algorithm 1.

The condition in the if statement in this routine can be checked in $\mathcal{O}(|X|)$ steps, so the for loop can be done in $\mathcal{O}(|X||\mathcal{R}|)$ time. In the worst case only one element from $\mathcal{R}$ is removed in each complete iteration of the for loop, so at most $|\mathcal{R}|$ iterations are necessary (possibly over multiple calls to ReduceToRA). Therefore, the overall running time of (possibly repeatedly) calling ReduceToRA is $\mathcal{O}\left(|X||\mathcal{R}|^{2}\right)$.

The second subroutine, ComputeClosure, computes the closure of $F$ relative to the current set of reactions $\mathcal{R}$, and is given in Algorithm 2. In this routine too, 


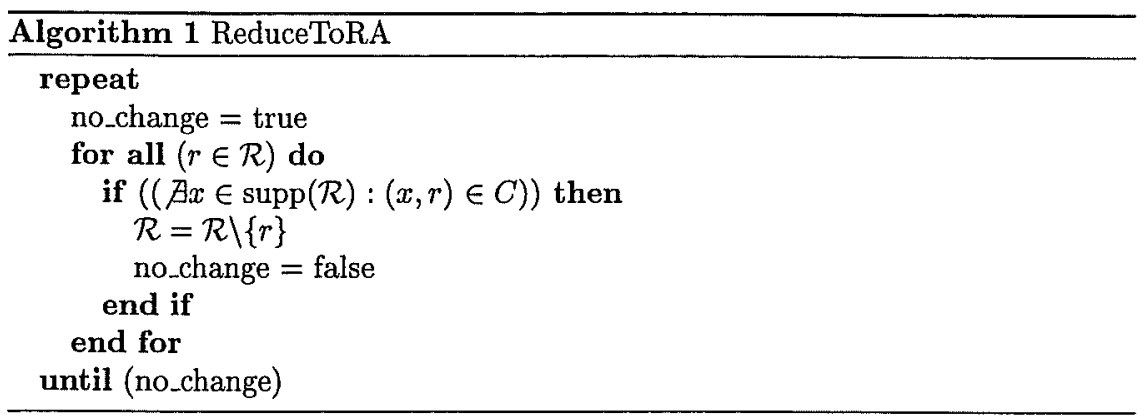

the condition in the if statement can be checked in $\mathcal{O}(|X|)$ steps, so the for loop can be done in $\mathcal{O}(|X||\mathcal{R}|)$ time. However, this might have to be done a total of $|\mathcal{R}|$ times (in the worst case), so the running time of one call to ComputeClosure is $\mathcal{O}\left(|X||\mathcal{R}|^{2}\right)$. The total number of calls to ComputeClosure is $\mathcal{O}(|\mathcal{R}|)$ (see below), so the overall running time of (possibly repeatedly) calling it is $\mathcal{O}\left(|X||\mathcal{R}|^{3}\right)$.

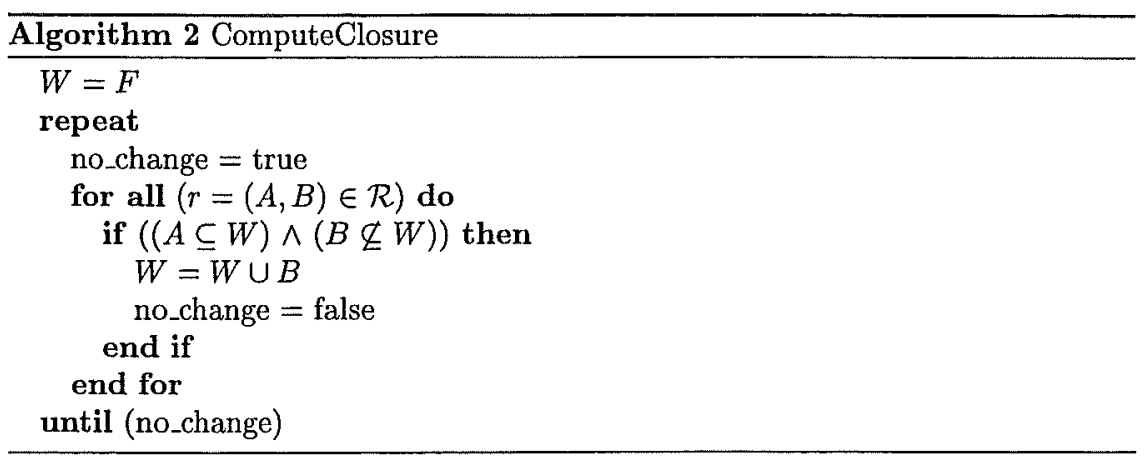

The third subroutine, ReduceToF-generated, implements the $\delta(\mathcal{R})$ function, and reduces a given reaction set $\mathcal{R}$ to a subset that is $\mathrm{F}$-generated (using the set $W$ as computed in the previous subroutine). In other words, the ReduceT'oF-generated routine repeatedly applies the reduction rule $\mathrm{R} 2$ until no more reductions can be made. The pseudocode for this routine is given in Algorithm 3.

Again, the condition in the if statement can be checked in $\mathcal{O}(|X|)$ steps, and so the for loop can be done in $\mathcal{O}(|X||\mathcal{R}|)$ time. The total number of calls to ReduceToF-generated is again $\mathcal{O}(|\mathcal{R}|)$, so the overall running time of (possibly repeatedly) calling this routine is $\mathcal{O}\left(|X||\mathcal{R}|^{2}\right)$

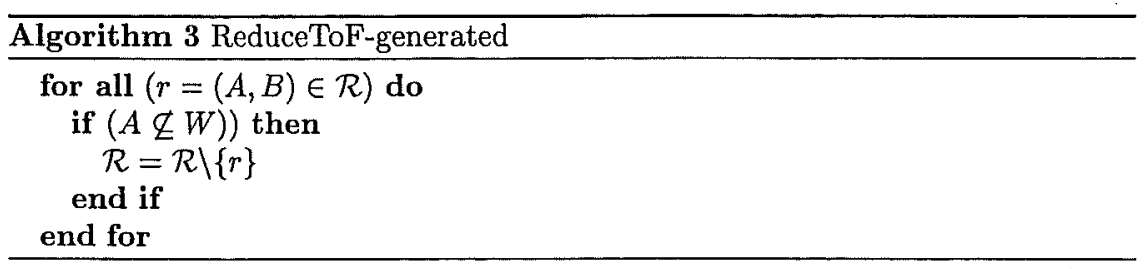


To run the actual RAF algorithm, first the ReduceToRA subroutine is called to reduce the set $\mathcal{R}$ to an RA set. Then ComputeClosure is called to compute the closure $W$ of $F$ relative to the (reduced) set $\mathcal{R}$. Finally, ReduceToF-generated is called to reduce $\mathcal{R}$ even further so that only reactions remain of which the reactants can be generated from the food set $F$. However, at this point, it is not guaranteed that $\mathcal{R}$ is still an RA set, so the three subroutines are called in sequence again, and again, until either no reactions are left or no more reductions can be made. From this, it follows that the total number of calls to the three routines is at most $|\mathcal{R}|$ (or $\mathcal{O}(|\mathcal{R}|)$ in general), since in the worst case only one reaction is removed from $\mathcal{R}$ each time.

The overall running time $\tau$ of the complete RAF algorithm is thus simply the sum of the overall running times of the three subroutines: $\tau=\mathcal{O}\left(|X||\mathcal{R}|^{2}\right)+$ $\mathcal{O}\left(|X||\mathcal{R}|^{3}\right)+\mathcal{O}\left(|X||\mathcal{R}|^{2}\right)=\mathcal{O}\left(|X||\mathcal{R}|^{3}\right)$. However, this is the absolute worst case (when at each calling sequence only one reaction is removed from $\mathcal{R}$ ), and will on average be better than this. Furthermore, using clever data structures (such as hash tables and ordered lists), the running time can be reduced even more, probably down to $\tau=\mathcal{O}\left(|R|^{2} \log |R|\right)$ (worst case). In Section 9 it is shown that the (average) running time of the algorithm is indeed much faster than this worst case upper bound.

\section{RANDOM CATALYTIC REACTION SYSTEMS}

In this section we will consider catalytic reaction systems $\mathcal{Q}=(X, \mathcal{R}, C)$ over $F$ for which $X, F$ and $\mathcal{R}$ are fixed, but $C$ is a random set of catalysations. In particular, we will suppose that each pair $(x, r) \in X \times \mathcal{R}$ is independently placed in $C$ with the same probability $p$. Under this model, we will let $\mathbb{P}(\mathcal{Q}, p)$ denote the probability that there exists an RAF set for $\mathcal{Q}$.

Proposition 8.1. $\mathbb{P}(\mathcal{Q}, p)$ is a monotone increasing function of $p$.

Proof. First note that if $C_{1} \subset C_{2} \subseteq X \times \mathcal{R}$, and if $\left(X, \mathcal{R}, C_{1}\right)$ contains an RAF set, then $\left(X, \mathcal{R}, C_{2}\right)$ contains an RAF set (by Lemma 4.4). Proposition 8.1 now follows by a straightforward application of the general monotonicity argument described in [2] (pp.36-37).

In Kauffman's original model [7, 8], $X=X(n)$ is the set of all possible sequences up to a given length $n$ over a $k$-letter alphabet $\{0,1, \ldots k-1\}$, and $F$ denotes all sequences of length $\leq t$ for some small but fixed value of $t$ (for example $t=2$ ). Actually, Kauffman considered in detail only the case where $k=2$, but we will consider the more general case as the calculations are similar. Also, Kauffman presents his model mostly in terms of 'protein-like polymers', but notes that it applies to the more general case of any kind of molecules. Hence, we will use the term 'molecules' here, to capture this broader range of the model.

Following $[7,8]$, the elements of $X(n)$ are regarded as oriented, and the set $\mathcal{R}=\mathcal{R}(n)$ of allowable reactions (representing ligation/cleavage reactions) is the set of reactions of the form $r=(\{a, b\},\{c\})$ and $r=(\{c\},\{a, b\})$ where $a, b, c \in X(n)$ 
for which $c=a b$ or $c=b a$, where $a b$ is the concatenation of $a$ with $b$ (in case $a=b$, $c$ is the concatenation of $a$ with itself). Thus, in Kauffman's original model, all reactions are bi-directional.

Finally, the set $C(n)$ of catalysations is randomly generated, by assigning elements of $X(n) \times \mathcal{R}(n)$ as follows: each $x \in X(n)$ catalyses any given reaction $r \in \mathcal{R}(n)$ with probability $p(n)$ (not dependent on $x$ or $r$ ), and these assignments are made independently over $X(n) \times \mathcal{R}(n)$ (a more realistic extension would allow catalysation probabilities to depend on lengths of molecules, but we don't explore this here). Let $f(n)$ denote the expected number of reactions that each molecule catalyses - thus

$$
f(n)=p(n)|\mathcal{R}(n)|
$$

Let $\mathcal{Q}(n)=(X(n), \mathcal{R}(n), C(n))$. An example of an irreducible RAF set for $\mathcal{Q}(5)$ (with $k=2$ and $t=2$ ) is shown in Fig. 3.

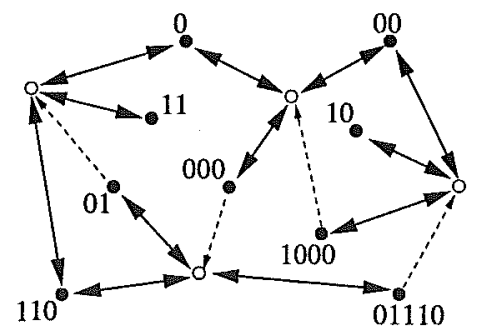

Figure 3. An irreducible RAF set for $\mathcal{Q}(5)$ with $k=2$ and $t=2$.

In this setting, two questions of interest include:

(1) Let $P_{\infty}=\lim _{n \rightarrow \infty} \mathbb{P}(\mathcal{Q}(n), p(n))$. Under what conditions on $f(n)$ (or, equivalently, on $p(n))$ is $P_{\infty}=1$ ? More generally when, and how, does the transition from $P_{\infty}=0$ to $P_{\infty}=1$ occur as the growth rate of the function $f$ increases?

(2) For any function $f$ for which $P_{\infty}=1$, at what value of $n$ will we expect to first observe at least one RAF set for $\mathcal{Q}(n)$, and how large (in terms of the number of reactions) will irreducible RAF sets typically be?

The following theorem, from [12], provides a partial answer to the first question (the first part of this theorem generalizes an earlier result from Kauffman [7, 8]).

Theorem 8.2 ([12]).

(i) If $f(n) \geq c n^{2}$ where $c>\log _{e}(k)$, then $P_{\infty}=1$.

(ii) If $f(n)<\frac{1}{3} e^{-1}$, then $P_{\infty}=0$.

In fact, under the condition described in part (i) of this theorem, the probability that $\mathcal{R}(n)$ is itself an RAF set for $\mathcal{Q}(n)$ tends to 1 as $n$ grows. The determination of $\mathcal{P}_{\infty}$ in the region between the remainder of $f(n)=O(1)$ and $f(n)=o\left(n^{2}\right)$ was not resolved in [12] but it was conjectured that when $f(n)=O(1)$ then $P_{\infty}=0$ 
and that for some function $f(n)=o\left(n^{2}\right)$ one has $P_{\infty}=1$. Support for the second part of this conjecture is provided by the following result (particularly, part (ii)).

\section{Theorem 8.3.}

(i) If $f(n) \geq d$ then $\operatorname{Pr}[\mathcal{Q}(n)$ has an $R A$ set $] \geq 1-e^{-2 d}$.

(ii) Suppose that $f(n) \geq d^{\prime} n \log (n)$ where $d^{\prime}>4$. Then, as $n \rightarrow \infty$, the expected number of $R A F$ sets for $\mathcal{Q}(n)$ tends to infinity.

Proof. Let $N(n)=|\mathcal{R}(n)|$. For any set $\mathcal{R}^{\prime}$ the probability that any particular reaction $r \in \mathcal{R}^{\prime}$ is catalysed by at least one molecule in $\operatorname{supp}\left(\mathcal{R}^{\prime}\right)$ is precisely

$$
1-(1-p(n))^{s\left(\mathcal{R}^{\prime}\right)}=1-\left(1-\frac{f(n)}{N(n)}\right)^{s\left(\mathcal{R}^{\prime}\right)}
$$

where $s\left(\mathcal{R}^{\prime}\right):=\left|\operatorname{supp}\left(\mathcal{R}^{\prime}\right)\right|$. Thus we obtain the following expression for the probability that $\mathcal{R}^{\prime}$ is an RA set for $\mathcal{Q}(n)$ :

$$
\operatorname{Pr}\left[\mathcal{R}^{\prime} \text { is an RA set for } \mathcal{Q}(n)\right]=\left(1-\left(1-\frac{f(n)}{N(n)}\right)^{s\left(\mathcal{R}^{\prime}\right)}\right)^{\left|\mathcal{R}^{\prime}\right|} \text {. }
$$

Applying the inequality $(1-x)^{y} \leq e^{-x y}$ for $x, y>0$ to this last equation gives:

$$
\operatorname{Pr}\left[\mathcal{R}^{\prime} \text { is an RA set for } \mathcal{Q}(n)\right] \geq\left(1-\exp \left(\frac{-f(n) s\left(\mathcal{R}^{\prime}\right)}{N(n)}\right)\right)^{\left|\mathcal{R}^{\prime}\right|} \text {. }
$$

We will use (4) and (5) to establish the two parts of the theorem.

Part (i) We show that $1-e^{-2 d}$ is a lower bound to the probability that $\mathcal{Q}(n)$ has an RA set of size 1. For each $r \in \mathcal{R}$, let $E_{r}$ be the event that $\{r\}$ is not an RA set for $\mathcal{Q}(n)$. Then, by (4),

$$
\operatorname{Pr}\left[E_{r}\right]=\left(1-\frac{f(n)}{N(n)}\right)^{s(\{r\})} \leq\left(1-\frac{d}{N(n)}\right)^{2}
$$

since $f(n) \geq d$ and $s(\{r\}) \geq 2$. The events $E_{r}$ are independent and so

$$
\operatorname{Pr}\left[\bigcap_{r \in \mathcal{R}} E_{r}\right]=\prod_{r \in \mathcal{R}} \operatorname{Pr}\left[E_{r}\right] \leq\left(1-\frac{d}{N(n)}\right)^{2 N(n)} .
$$

Finally, by the inequality $\left(1-\frac{x}{N}\right)^{N} \leq e^{-x}$ for $x>0$ we have

$$
\operatorname{Pr}[\mathcal{Q}(n) \text { has an RA set of size } 1]=1-\operatorname{Pr}\left[\bigcap_{r \in \mathcal{R}} E_{r}\right] \geq 1-e^{-2 d}
$$

as required.

Part (ii) We will use the notation $f_{1}(n) \sim f_{2}(n)$ when $\lim _{n \rightarrow \infty} \frac{f_{1}(n)}{f_{2}(n)}=1$. From [12](Equation 2), $|\mathcal{R}(n)| \sim n|X(n)| \sim n 2^{n+1}$ and so, if we let $h(n):=\frac{n 2^{n+1}}{N(n)}$ then we have

$$
h(n) \sim 1 .
$$


Let $\Delta X(n):=X(n)-X(n-1)$, the set of binary sequences of length exactly $n$, and let

$$
W(n):=\left\{X^{\prime} \subseteq \Delta X(n):\left|X^{\prime}\right|=2^{n-1}\right\} .
$$

Thus $X^{\prime} \in W(n)$ precisely if $X^{\prime}$ consists of exactly half of the possible binary sequences of length $n$.

For each $X^{\prime} \in W(n)$ define $S\left(n, X^{\prime}\right)$ and $\mathcal{R}\left(n, X^{\prime}\right)$ to be the minimal subsets of $X(n)$ and $\mathcal{R}(n)$ respectively that satisfy the conditions

$$
X^{\prime} \subseteq S\left(n, X^{\prime}\right) \text {, and }
$$

$c \in S\left(n, X^{\prime}\right), c=a b \Rightarrow a, b \in S\left(n, X^{\prime}\right)$, and $(\{a, b\},\{c\}),(\{c\},\{a, b\}) \in \mathcal{R}\left(n, X^{\prime}\right)$.

Note that the map from $W(n)$ to $\mathcal{R}(n)$ defined by

$$
X^{\prime} \mapsto \mathcal{R}\left(n, X^{\prime}\right)
$$

is one-to-one and that

$$
\left|\operatorname{supp}\left(R\left(n, X^{\prime}\right)\right)\right|=\left|S\left(n, X^{\prime}\right)\right| \geq\left|X^{\prime}\right|=2^{n-1}
$$

Furthermore, for any $X^{\prime} \in W(X), R\left(n, X^{\prime}\right)$ is $F$-generated, by virtue of the way that $\mathcal{R}\left(n, X^{\prime}\right)$ is constructed from $X^{\prime}$. Thus, $\mathcal{R}\left(n, X^{\prime}\right)$ is an RAF set for $\mathcal{Q}(n)$ if and only if $\mathcal{R}\left(n, X^{\prime}\right)$ is an RA set for $\mathcal{Q}(n)$. For any given set $X^{\prime} \in W(n)$ we may apply inequality (5), together with the inequality $f(n) \geq d^{\prime} n \log (n)$ and (7) to deduce that the probability that $\mathcal{R}\left(n, X^{\prime}\right)$ is an RA set for $\mathcal{Q}(n)$ is at least

$$
\left(1-\exp \left(\frac{-d^{\prime} n \log (n) 2^{n-1}}{N(n)}\right)\right)^{\left|\mathcal{R}\left(n, X^{\prime}\right)\right|}
$$

Thus, since $\left|\mathcal{R}\left(n, X^{\prime}\right)\right| \leq|\mathcal{R}(n)|=N(n)$, and by the definition of $h$ we have

(8) $\operatorname{Pr}\left[\mathcal{R}\left(n, X^{\prime}\right)\right.$ is an RAF set for $\left.\mathcal{Q}(n)\right] \geq\left(1-\exp \left(\frac{-d^{\prime} h(n) \log (n)}{4}\right)\right)^{N(n)}$.

Let $q(n)$ denote the right-hand term in (8), which we may re-write as

$$
q(n)=\theta_{n}^{2^{n}}
$$

where $\theta_{n}=\left(1-n^{-d^{\prime} h(n) / 4}\right)^{2 n / h(n)}$. Since $d^{\prime}>4$ and $h(n) \sim 1$ (from (6)) it is easily checked that

$$
\theta_{n} \sim 1
$$

Now,

$$
|W(n)|=\left(\begin{array}{c}
2^{n} \\
2^{n-1}
\end{array}\right) \sim c^{\prime} \frac{2^{2^{n}}}{2^{n / 2}}
$$

where $c^{\prime}>0$ is a constant. Combining (9) and (11) gives

$$
|W(n)| q(n) \sim c^{\prime} \frac{\left(2 \theta_{n}\right)^{2^{n}}}{2^{n / 2}}
$$

Let $\mu^{\prime}(n)$ be the expected number of subsets of $\mathcal{R}(n)$ that are an RAF set for $\mathcal{Q}(n)$. Since the mapping $X^{\prime} \mapsto \mathcal{R}\left(n, X^{\prime}\right)$ is one-to-one, it follows that

$$
\mu^{\prime}(n) \geq \sum_{X^{\prime} \in W(n)} \operatorname{Pr}\left[\mathcal{R}\left(n, X^{\prime}\right) \text { is an RAF set for } \mathcal{Q}(n)\right] .
$$


Combining this inequality with (8), we see that $\mu^{\prime}(n)$ is asymptotically bounded below by $|W(n)| q(n)$, and this latter function tends to infinity as $n$ grows (by (10) and (12)) so that $\mu^{\prime}(n)$ tends to infinity with $n$ as well. This completes the proof.

\section{Simulations}

We have implemented the RAF algorithm and used it on Kauffman's original model to study the questions raised in the previous section numerically. Even though our algorithm is polynomial in $|\mathcal{R}|$ (the total number of reactions), in Kauffman's model this number increases exponentially with increasing maximum molecule length $n$, so the values for $n$ that can be numerically investigated in a reasonable time is still rather limited. In the results presented in this section, the highest value for $n$ used is 20 . The data shown are averages over 100-10000 randomly generated instances of the Kauffman model (more instances for smaller values of $n$, fewer for larger values). In all simulations, we used $k=2$ (the 'molecules' are all possible bit strings up to length $n$ ), and $t=2$, so $F=\{0,1,00,01,10,11\}$.

Figure 4 shows the observed probabilities and relative sizes of RA(F) sets for a range of $p(n)$ values for various $n$. The graph on the left shows the probability $\operatorname{Pr}[\mathrm{RAF}]$ of the occurrence of an $\mathrm{RA}(\mathrm{F})$ set against $f(n)=p(n)|\mathcal{R}(n)|$ in the range $[0 ; 2.5]$. The graph on the right shows the (average) relative size $\left|\mathcal{R}^{\prime}\right| /|\mathcal{R}|$ of an $\mathrm{RA}(\mathrm{F})$ set $\mathcal{R}^{\prime}$ compared to the complete set $\mathcal{R}$. Note that the sizes of the RA sets are only a small fraction of $|\mathcal{R}|$, and only the data for $n=15,20$ are actually visible.
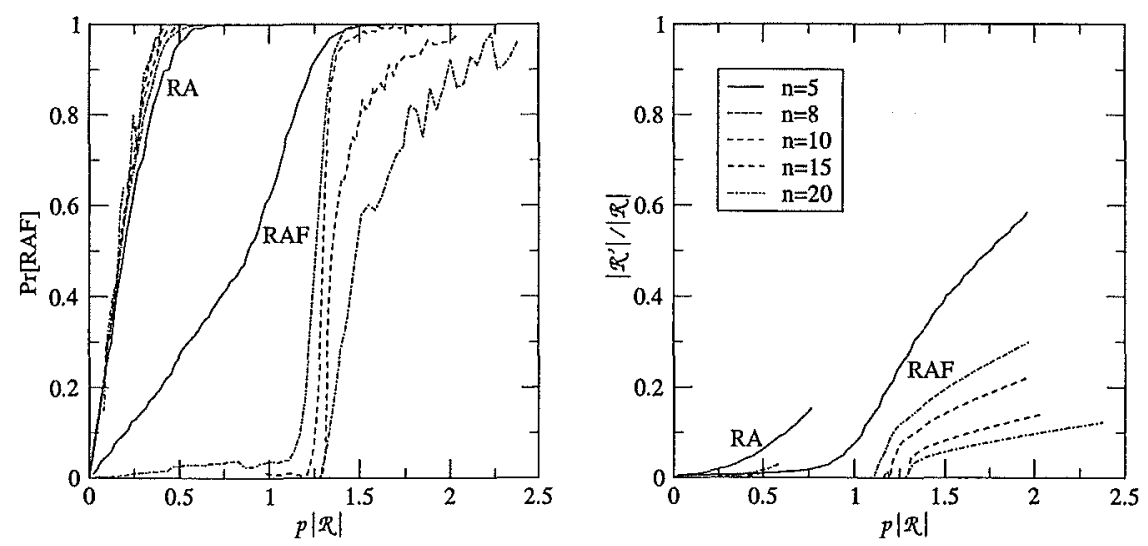

FIgURE 4. The probability of occurrence (left) and the relative size (right) of RA(F) sets in Kauffman's model.

As the graph clearly shows, for small values of $p(n)$ (in particular such that $f(n)<1$ for $n>5$ ), the probability of an RAF set occurring is negligible, which, according to theorem $8.2(\mathrm{i})$, is to be expected. Then there is a sharp transition, roughly between $1.25 \leq f(n) \leq 1.5$, after which $\operatorname{Pr}[\mathrm{RAF}]$ converges to 1 . However, the transition appears to become less sharp and less smooth for the larger values 
of $n$ (although this is partly caused by the fewer instances over which the results are averaged, resulting in a somewhat less accurate average).

The average size of an RAF set increases slowly with increasing $p(n)$. Initially, once the probability of having an RAF set becomes positive, the RAF sets are still very small (often of size $\mathcal{O}(1)$, e.g., a reaction that has two elements from the food set as its reactants, and which is catalysed by one of the molecules involved in the reaction itself). They then grow quickly in size during the transition phase, but only slowly (and seemingly almost linearly) afterwards. Eventually, as $p(n)$ becomes large enough, the entire set $|R|$ will be one large (maximal) RAF.

Figure 5 shows the observed (average) relative size $\left|i \mathcal{R}^{\prime}\right| /\left|\mathcal{R}^{\prime}\right|$ of an irreducible $\mathrm{RAF}$ set $i \mathcal{R}^{\prime}$ compared to the maximal RAF set $\mathcal{R}^{\prime}$ it was derived from. Initially, when the maximal RAF sets are still small themselves, they cannot be reduced any further, and thus the irreducible RAF sets will be about as large as the maximal RAF sets themselves. But as the size of a maximal RAF sets grows, it is more likely that it will consist of several (unconnected) irreducible ones. This graph also clearly shows the transition range $1.25 \leq f(n) \leq 1.5$, during which the relative size of an irreducible RAF decreases quickly, and after which it decreases only very slowly.

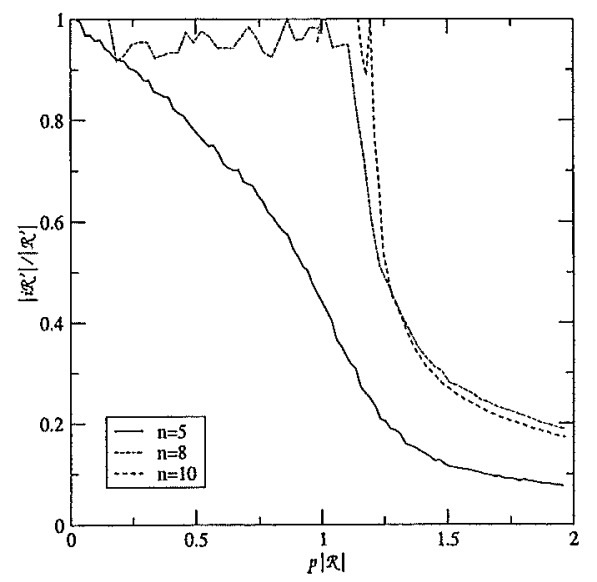

Figure 5. The (average) relative size of an irreducible RAF set.

An important, but as yet analytically unanswered question is how the transition point scales with $n$. Does it increase without limit (and if so, how), or does it converge to some finite value? Figure 6 shows the transition points plotted against $n$. The black circles represent the values for $f(n)=p(n)|\mathcal{R}(n)|$ (averaged over 1000 random instances) for values of $p(n)$ for which $\operatorname{Pr}[\mathrm{RAF}]$ is around 0.5 , for $n=7,8, \ldots, 20$. The black squares show the exact values of Pr[RAF] for which the data was taken for each value of $n$. At least up to $n=20$, it appears that the transition point scales linearly with $n$. The solid black line represents a linear fit: $l(n)=1.107+0.018 n$. The dashed line shows the $\operatorname{Pr}[\mathrm{RAF}]=0.5$ reference point. 


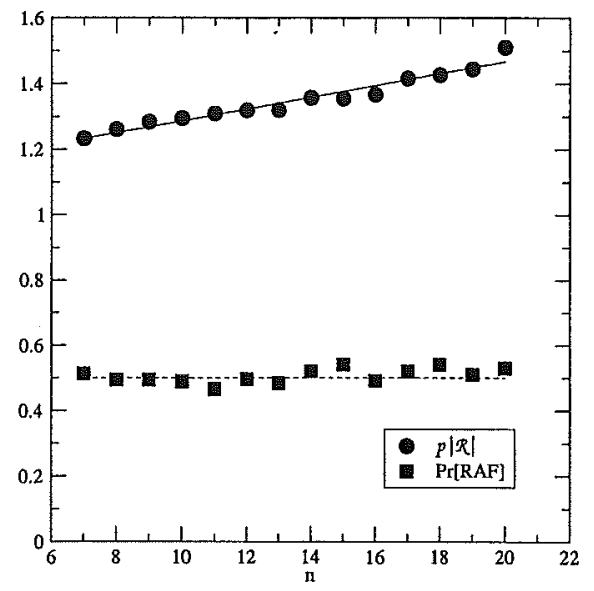

FIgURE 6. The transition points as a function of $n$.

To check the average running time of our implementation of the RAF algorithm, figure 7 shows the actual running times (black circles; in CPU seconds) needed to generate the data of figure 6 . As the figure shows, the data follows a straight line quite well. The solid line in the plot represents a fitted power law: $\tau=0.0002|\mathcal{R}|^{1.43}$. So, in fact, our implementation has a sub-quadratic (average) running time (i.e., much better than the analytical cubic worst case running time derived in section 7 ), and with a very small constant.

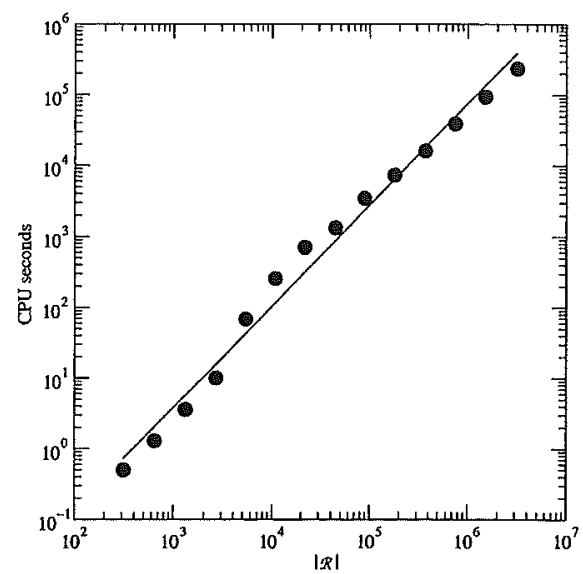

FIGURE 7. Actual running time of our implementation of the RAF algorithm on Kauffman's model. 


\section{Conclusions}

We have described a polynomial-time algorithm for determining whether an arbitrary catalytic reaction system contains an RAF set, without having to search through the exponentially large space of all possible subsets of reactions. The algorithm relies on some simple combinatorial properties of catalytic reaction systems, and the techniques described may be useful for investigating a variety of random biochemical models.

In this paper we have demonstrated the algorithm's use for the Kauffman molecule model, and indeed our simulations would not have been possible without this algorithm for even modest values of $n$ (eg. $n=10$ ). For the Kauffman model, one particular question we considered was the degree of catalysation (as measured by $f(n)$ ) required for the likely emergence of an RAF set, and more particularly, how this depends on the lengths $(n)$ of the molecules. Our analytic and simulation results suggest that the growth rate of $f(n)$ required for the likely appearance of an RAF set is sub-quadratic in $n$, and apparently close to linear, in line with an earlier conjecture from [12]. In this sub-quadratic region the probability that any given set is an RAF set tends quickly to 0 with increasing $n$, yet the very large number of possible subsets ensures that at least one of them is RAF.

There is clearly the possibility for further analytic work to provide more precise results for the Kauffman model, as well as for extensions to it - for example to models in which the probability that a molecule catalyses a reaction is dependent on the molecule's length. It may also be interesting to explore the dynamical aspects of these models, where catalysed reactions are accelerated, and there are many copies of each molecule (with an unlimited food source), as well as some competition for space (for example, induced by removing excess molecules). In this setting it would be interesting to see if RAF sets tend to become prominent.

Furthermore, out RAF algorithm is likely to be useful in other settings as well. One example is to take a given large set of real biochemical reactions and then to assign catalysations randomly (according to some stochastic scheme, perhaps reflecting the actual average rate of catalysation) and to determine the probability that the system contains an RAF. In this way it may be possible to shed some light on the question of whether autocatalytic, self-sustaining systems rely on a very special and unlikely biochemical balance, or whether they are a likely consequence of a sufficiently complex system of biochemical reactions. Another example is reaction sets evolved by simple computer programs. Using a genetic algorithm, artificial reaction sets have been evolved successfully to generate pre-specified molecule distributions $[10,6]$. Our RAF algorithm can be applied directly to such evolved reaction sets to investigate the emergence of autocatalytic (RAF) sets, a property that is often believed to be important in reaching the pre-specified molecule distributions. 


\section{ACKNOWLEDGEMENTS}

We thank the Allan Wilson Centre for Molecular Ecology and Evolution for allowing us to use their Beowulf cluster Helix for our numerical simulations. Also thanks to David Penny for helpful comments.

\section{REFERENCES}

[1] Bollobas, B. \& Rasmussen, S. (1989). First cycles in random directed graph processes. Discr. Math. 75, 55-68.

[2] Bollobas, B. (2001). Random Graphs (2nd ed.). Cambridge studies in advanced mathematics vol. 73. Cambridge University Press.

[3] Bonchev D. \& Mekenyan O. (1994). Graph theoretical approaches to chemical reactivity. Kluwer.

[4] Cohen, J. E. (1988). Threshold phenomena in random structures. Discr. Appl. Math. 19, 113-128.

[5] Dyson, F. (1985). Origins of Life. Cambridge University Press.

[6] Hordijk, W. \& Fontanari, J. F. (2003). Catalytic reaction sets, decay, and the preservation of information. To appear in KIMAS '03.

[7] Kauffman, S. A. (1986). Autocatalytic sets of proteins. J. theor. Biol. 119, 1-24.

[8] Kauffman, S. A. (1993). The origins of order: self-organization and selection in evolution. Oxford University Press.

[9] Lifson, S. (1997). On the crucial stages in the origin of animate matter. J. Mol. Evol. 44, $1-8$.

[10] Lohn, J, D., Comombano, S. P., Scargle, J., Stassinopoulos, D. \& Haith, G. L. (1998). Evolving catalytic reaction sets using genetic algorithms. IEEE International Conference on Evolutionary Computation.

[11] Joyce, G. F. (1989). RNA evolution and the origin of life. Nature 338, 217-224

[12] Steel, M. (2000). The emergence of a self-catalysing structure in abstract origin-of-life models. Applied Mathematics Letters 3, 91-95. 